\title{
Competencias socioemocionales en la identidad profesional de los profesores en formación
}

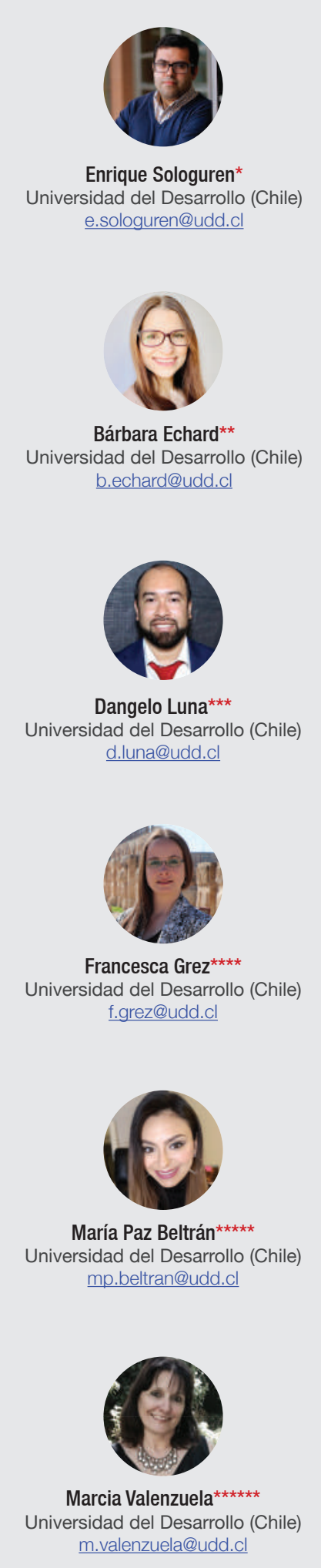

\section{Socio-Emotional Competencies In Pre- Service Teachers' Professional Identity}

Recibido: 2 de julio de 2021 | Aprobado: 20 de agosto de 2021

\section{Resumen}

Las competencias socioemocionales son un elemento clave en la formación docente. En este contexto, el objetivo es analizar las percepciones sobre estas competencias de los profesores en formación en la construcción de la identidad profesional. La metodología es mixta de tipo secuencial exploratorio, transversal y con alcance descriptivo. El componente cualitativo recurre al análisis documental y a las entrevistas en profundidad para identificar y categorizar las percepciones sobre las habilidades socioemocionales. El componente cuantitativo se desarrolla mediante la aplicación y análisis de la escala de Rasgo de Metaconocimiento de los Estados Emocionales validada para el español y administrada a la totalidad de los estudiantes de la cohorte 2020. Los resultados, obtenidos a partir de información concurrente, indican que estas competencias están asociadas con experiencias y creencias diversas, las que facilitan u obstaculizan el desempeño profesional en el aula. Implicancias para las trayectorias estudiantiles son discutidas.

Palabras clave: Competencias socioemocionales, identidad profesional, formación inicial docente, programa de formación pedagógica para licenciados.

\footnotetext{
* Doctor en Lingüística por la Pontificia Universidad Católica de Valparaíso, Chile.

** Magíster en Educación en Segunda Lengua por la Universidad de Toronto, Canadá.

*** Magíster en Educación mención en Evaluación de aprendizajes por la Pontificia Universidad Católica de Chile.

**** Magíster en Historia por la Universidad de Chile.

***** Magíster en Didáctica de las Ciencias Experimentales por la Pontificia Universidad Católica de Valparaíso.

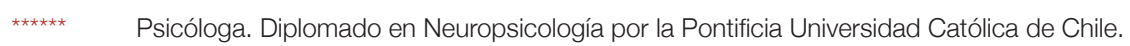

ISSN (en línea): 1814-4152 / Sitio web: http://cuaderno.pucmm.edu.do

Cómo CITAR: Sologuren, E., Echard, B., Luna, D., Grez, F., Beltrán, M. P. y Valenzuela, M. (2022). Competencias socioemocionales en la identidad profesional de los profesores en formación. Cuaderno de Pedagogía Universitaria, 19 (37), 115-132. 


\section{Abstract}

Socio-emotional competencies are a crucial element in teaching training. In this context, the aim is to analyze pre-service teachers' perceptions about these competencies in the process of building their professional identities. A mixed methodology was used with a particular focus on sequential exploratory and descriptive techniques. The qualitative component considers documentary analysis and in-depth interviews to identify and categorize the perceptions about socioemotional skills. The quantitative component was carried out by applying and analyzing the Trait Meta-Mood Scale (TMMS24) test -in its validated version in Spanish- and administered to all pre-service teachers of the 2020 cohort. The results obtained from concurrent data point out that socio-emotional competencies may be linked to diverse experiences and beliefs that could either foster or hinder professional performance in the classroom. Implications for teacher training are further discussed.

Keywords: Socio-emotional competencies, professional identities, teacher education, teacher training program.

\section{Introducción y marco referencial}

La identidad docente se construye, individual y colectivamente, durante el proceso formativo, especialmente en el desarrollo de las asignaturas referidas al posicionamiento didáctico y disciplinario. Asimismo, los factores individuales como las emociones, valores, experiencias, y motivaciones juegan un rol clave en esta construcción. Sin embargo, los docentes en formación pueden encontrar obstáculos y desafíos al momento de enfrentarse a la realidad educativa en los centros de práctica, lo que en ocasiones los desanima, llegando a cuestionar su propia identidad profesional. Desde esta perspectiva, es necesario incorporar en los procesos formativos las competencias socioemocionales (CSE), dado que permiten el autoconocimiento emocional y social, dimensiones fundamentales para generar una identidad docente sólida.

La investigación que reportamos fue realizada en un Programa de Formación Pedagógica (PFP) para Licenciados en una universidad privada chilena. El propósito ha sido contribuir a la formación inicial docente de los profesores en formación del Programa a través de la exploración de la construcción de identidad profesional y su vínculo con las CSE.
1.1 Noción de competencia socioemocional En un primer momento emerge el concepto de Inteligencia Emocional (IE) en las publicaciones del grupo de Salovey y Mayer (1995). Esta se refiere a la capacidad de razonar y resolver problemáticas asociadas a las emociones e identificar su significado (Mayer et al., 2011). En esta línea, Álvarez et al. (2019) midieron la autopercepción de la IE como aproximación a las CSE reportadas por profesores en formación en un programa de pedagogía de una universidad chilena, utilizando el instrumento propuesto por estos autores, y que mide las dimensiones de Atención (habilidad de percibir adecuadamente las emociones), Claridad (comprender las emociones propias) y Reparación (la regulación de las emociones de forma apropiada). A esto se pueden sumar otras dimensiones para abordar el constructo, como son la comunicación efectiva, resolución de conflictos, empatía, toma de decisiones responsable, entre otras, que se han reportado como relacionadas con prácticas de enseñanza eficaces y bienestar para estudiantes y profesores (Hirshberg et al., 2020).

Para efectos de este trabajo de investigación se mencionan como sinónimos los términos de competencia socio-emocional y competencia emocional (o competencias socio-emocionales), al margen de la existencia de las legítimas discrepancias que pueden emanar desde los 
expertos en el campo de la Psicología. Por su parte, dado el solapamiento existente de estos términos con el concepto de IE, hemos decidido emplearlo como indicador que revelaría aquellos aspectos de las CSE de nuestro interés. Los investigadores Álvarez et al. (2019) midieron la autopercepción de la IE como aproximación a las CSE reportadas por los profesores en formación de un programa de pedagogía de una universidad chilena. En este caso, la IE se midió en los aspectos de Atención, Claridad y Reparación, donde la primera se define como la habilidad de percibir adecuadamente las emociones; así, la segunda corresponde a comprender las emociones propias; y la última, que consiste en la regulación de las emociones de forma apropiada (Fernandez-Berrocal, Extremera y Ramos, 2004). En el presente estudio, se utilizarán estas dimensiones para explorar el constructo de la competencia socio emocional del contexto situado del Programa de Formación Pedagógica.

\subsection{Las competencias socioemocionales en la formación de profesores y construcción de identidad}

\subsubsection{Importancia en la formación del profesorado}

Existe cierto consenso en cuanto al rol de las CSE en la formación de la identidad docente y cómo éstas tributarían en docentes capaces de modelar estas competencias para facilitar el desarrollo de sus jóvenes estudiantes, y, por lo tanto, se vislumbra en la literatura una orientación hacia la enseñanza explícita de estas en programas de formación docente. Se reconoce que los profesores con CSE desarrolladas se relacionan mejor con los integrantes de la comunidad escolar y pueden aportar a la formación de las mismas en sus propios estudiantes (Bisquerra, 2019; Nenonene et al., 2019). Por lo tanto, para lograr adquirir estas habilidades, los profesores en formación deben ser emocionalmente "alfabetizados" (Waajid et al., 2013). Por otra parte, incluir en la formación del profesorado las CSE podría apoyar su ingreso en el plano laboral. En esta línea, se sabe que aquellos docentes que son capaces de manejar las consecuencias negativas del estrés y con ello prevenir aquellas enfermedades asociadas, coinciden con un buen dominio de habilidades relacionadas a la IE (Extremera y Fernández-Berrocal, 2004). Adicionalmente, Pérez
(2003) señala que son diversos los trabajos que vinculan la IE y las CSE con el afrontamiento a situaciones estresantes, el desarrollo profesional y la satisfacción personal; elementos importantes para la vida de las personas. Asociado a lo anterior, Mearns y Cain (2003) afirman que aquellos docentes que se perciben a sí mismos con mayor $\mathrm{IE}$, presentan mayor ajuste emocional y un mayor uso de estrategias de supresión de pensamientos negativos (Cassullo y García, 2015).

\subsubsection{Competencias socioemocionales e Identidad Profesional}

Los aportes actuales de las neurociencias en el ámbito de la educación revelan que la estructura de nuestro cerebro, específicamente la corteza prefrontal, juega un rol esencial en la planificación y regulación de nuestras acciones, está articulada de modo tal que se integran los impulsos, las emociones y los pensamientos para poder dar una respuesta adaptativa a las diferentes situaciones que enfrentamos. En particular, el proceso de aprendizaje no es posible concebirlo sin esta integración tripartita, puesto que se estaría negando parte de nuestra estructura constitutiva. (Raspall, 2019). Estos nuevos e incipientes alcances en el conocimiento del cerebro, vienen a derribar algunos mitos tradicionales que impulsan una separación entre la emocionalidad y la racionalidad, aspectos que presentan una relación dialéctica indisoluble.

De ese modo, el presente artículo se adhiere a una corriente que entiende que los factores cognitivos y emocionales estarían involucrados en el proceso de construcción identitaria de los profesores. En esta línea, varios estudios concuerdan en que las emociones se vinculan con la identidad profesional docente (Schutz y Lee, 2014; O'Connor, 2008; Zembylas, 2003; Kimura 2010; Reio, 2005) pese a que difieren respecto a su predominancia o maneras de articulación. Ahora bien, en términos generales, los diversos trabajos desarrollados en torno a ello han planteado que las emociones permiten a los profesores en servicio dar sentido a las vivencias del contexto educativo. Además, permiten motivar a los docentes en sus tareas cotidianas, pues los impulsan, incentivan y estimulan a seguir actuando de una determinada manera. Independientemente 
de la corriente teórica, estos autores coinciden en que la identidad profesional se forja por medio de diversas experiencias y ámbitos. Tal como proponen Timostsuk y Ugaste (2010), la construcción de la identidad profesional es influenciada por aspectos profesionales tales como las remuneraciones, el ambiente laboral; aspectos personales como la edad, experiencia, motivación, estado físico, psicológico e intelectual; elementos contextuales como la familia y amigos; temas sociales como situaciones económicas, políticas y sociales, además de elementos vocacionales (Vieira, 2011).

Considerando lo anterior es que la propuesta elaborada por Mayer y Salovey cobra gran relevancia, pues la IE se consideraría como una habilidad centrada en el procesamiento de la información emocional que unifica las emociones y el razonamiento. Sería un proceso mediante el cual el individuo utiliza sus emociones para facilitar un razonamiento más efectivo y pensar de forma más inteligente sobre la vida emocional (Mayer y Salovey, 1997). Proceso que, Grewal y Salovey (2005) precisan, es independiente de los rasgos estables de personalidad. Por tanto, se puede enseñar, practicar y modificar a lo largo de la vida1.

En síntesis, si consideramos que las CSE son un elemento clave en la construcción de la identidad profesional docente, se desprende entonces la necesidad de que éstas formen parte de la formación inicial docente. Sin embargo, diversos estudios han demostrado que actualmente existe una carencia de estas en los programas de estudio (Bisquerra, 2019; Vivas et al., 2010; FragosoLuzuriaga, 2015) e investigaciones que persiguen medir las CSE en estudiantes de pedagogía son limitados. Experiencias como las de Corcoran y Tormey (2010) demostraron que en general la inteligencia emocional de los profesores en formación resulta ser más baja que el promedio de profesores en servicio, lo cual sugiere la necesidad de trabajar las CSE durante la formación. Por otro lado, intentos sistemáticos de incorporar las CSE de forma explícita en los currículos o planes de estudio de profesores en formación apuntan a implementar estrategias remediales o limitadas a cursos del programa, como la experiencia de Waajid et al. (2013) o bien, como es el caso descrito por Nenonene, et al. (2019) se encuentran en etapa incipiente.

\section{Propósito del estudio y pregunta de investigación}

En vista de todo lo anteriormente discutido, el presente trabajo comienza por formular la pregunta de investigación: ¿Cómo los profesores en formación del PFP perciben el rol de sus competencias socioemocionales en la construcción de la identidad profesional? El estudio tiene como objetivo general analizar las percepciones sobre las CSE de los profesores en formación del PFP en la construcción de la identidad profesional con el objeto de informar las trayectorias formativas. Para ello se utilizó un enfoque mixto o multi-método incorporando instrumentos cualitativos y cuantitativos. De este modo, y siguiendo lo planteado por Creswell (2018), la utilización del modelo mixto permitió obtener una mayor variedad de perspectivas del problema, particularmente referidos a frecuencia, amplitud y magnitud, así como profundidad y complejidad; generalización y comprensión de la percepción que los/as estudiantes del Programa asignan a las habilidades socioemocionales sobre su identidad profesional. Particularmente, se escogió un modelo de ejecución exploratorio secuencial (Creswell, 2018).

Con el objetivo de describir el contexto curricular de enseñanza-aprendizaje del programa PFP, se efectuó un análisis documental de los programas de estudio de la Carrera. Posteriormente, se aplicaron dos instrumentos de recolección de información para identificar las percepciones de los profesores en formación sobre las CSE. Primero se aplicó el Trait Meta-Mood Scale (TMMS24) (Álvarez, 2019), un instrumento de autorreporte individual respondido por todos los estudiantes de manera voluntaria de la cohorte 2020 y, en una segunda instancia, se desarrollaron entrevistas individuales a cinco estudiantes.

1 Para más información sobre ello se puede consultar el artículo de Fernández Berrocal y Extremera Pacheco (2005). 
Con el objetivo de describir el contexto curricular de enseñanza-aprendizaje del programa PFP, se efectuó un análisis documental de los programas de estudio de la Carrera. Posteriormente, se aplicaron dos instrumentos de recolección de información para identificar las percepciones de los profesores en formación sobre las CSE. Primero, se aplicó el Trait Meta-Mood Scale (TMMS24) (Álvarez, 2019), un instrumento de autoreporte individual respondido por todos los estudiantes de manera voluntaria de la cohorte 2020 y, en una segunda instancia, se desarrollaron entrevistas individuales a cinco estudiantes.

Este artículo está organizado del siguiente modo: una breve introducción en torno a la noción de CSE que sustentó la investigación como también su relación con el concepto de identidad profesional. En un segundo momento, se presenta el marco metodológico junto con el marco contextual del programa de estudio en el que se aplicaron los instrumentos. En tercer lugar, se presentan los resultados obtenidos después de la aplicación de los diversos procedimientos. Posteriormente, se verán las conclusiones que aparecen a partir de los hallazgos obtenidos.

\section{Metodología}

\subsection{Descripción del contexto PFP y objetivo}

Los participantes son 36 estudiantes del Programa de Formación Pedagógica (PFP), de la Universidad del Desarrollo en Chile. El programa está dirigido a profesionales que ya poseen un título válido en Chile y que desean obtener una segunda titulación como profesor de Educación Media, en diferentes menciones.

El curriculum de este programa considera: Las didácticas y las disciplinas de cada mención, y trece asignaturas generales para los estudiantes del programa ${ }^{2}$. Esta formación tiene una duración de un año y desde el comienzo los participantes realizan su práctica profesional.

El objetivo general es analizar las percepciones sobre las CSE de los profesores en formación del
PFP en la construcción de la identidad profesional con el objeto de informar las trayectorias formativas y los objetivos específicos: describir el contexto curricular de enseñanza-aprendizaje del programa PFP; identificar percepciones sobre las CSE de los profesores en formación del PFP; categorizar las percepciones (representaciones, teorías, supuestos, creencias) sobre las CSE de los profesores en formación en función de su rol en la construcción identitaria profesional; y proponer líneas de acción con respecto a las percepciones analizadas sobre las CSE para la toma de decisiones en el currículum.

\subsection{Tipo de investigación y diseño metodológico}

El diseño metodológico de esta propuesta es mixto, no experimental, con alcance exploratoriodescriptivo y temporalidad de corte transversal (Creswell y Creswell, 2018). El propósito de este enfoque es recolectar y analizar la información cuantitativa y cualitativa para elaborar un panorama más integral, completo y holístico (Mertens, 2019; Angouri, 2010; Creswell, 2013). De este modo, se logró una perspectiva más amplia y profunda del fenómeno estudiado: las percepciones de las competencias socioemocionales en los profesores en formación.

Este tipo de diseño metodológico contempla cuatro fases: la primera de naturaleza cualitativa con carácter exploratoria, la segunda de análisis cuantitativo, una tercera de tipo cualitativo y la cuarta de integración de datos obtenidos a partir de la aplicación de las diversas estrategias metodológicas. En una primera fase se recolectan y analizan datos cualitativos para explorar los antecedentes referidos a la presencia de las CSE en el PFP. En una segunda etapa se recolectan y analizan datos cuantitativos por medio de la aplicación de las encuestas para así obtener una base de datos. En tercer lugar, se aplicaron entrevistas a actores claves, lo que nos permitió construir una nueva base de datos que profundiza y amplía los resultados obtenidos a partir del análisis. Finalmente, los descubrimientos de ambas etapas

2 Las trece asignaturas comprendidas en dicho conjunto se refieren a los cursos de Formación Pedagógica General y Profesionalismo Docente entre los cuales se encuentran Evaluación, Currículum, Gestión de Aula, Fundamentos Filosóficos y Psicología, entre otros. Para más detalle revisar: https:// educacion.udd.cl/carrera/programa-formacion-pedagogica/malla-curricular 
se comparan e integran en la interpretación y elaboración de los resultados. En la siguiente tabla se presenta la relación entre objetivos, instrumentos, procedimientos y frecuencias de aplicación:

\section{Tabla 1.}

Aspectos metodológicos y su relación con los objetivos de investigación

\begin{tabular}{|c|c|c|c|}
\hline $\begin{array}{l}\text { Instrumento de } \\
\text { recolección }\end{array}$ & $\begin{array}{l}\text { Proceso } \\
\text { de análisis }\end{array}$ & $\begin{array}{l}\text { Frecuencia de } \\
\text { aplicación }\end{array}$ & Objetivo \\
\hline $\begin{array}{l}\text { Ficha de registro } \\
\text { y análisis curricular }\end{array}$ & $\begin{array}{c}\text { Análisis documental / } \\
\text { Interrogación al texto: } \\
\text { macroestructuras semánticas } \\
\text { (Van Dijk, 1983). }\end{array}$ & $\begin{array}{c}40 \text { Programas de estudios } \\
1 \text { Perfil de egreso }\end{array}$ & $\begin{array}{l}\text { Objetivo específico 1: } \\
\text { Describir el contexto curricular } \\
\text { de enseñanza-aprendizaje del } \\
\text { programa PFP, con énfasis en } \\
\text { el aula de didáctica-práctica }\end{array}$ \\
\hline $\begin{array}{l}\text { Instrumento de } \\
\text { autoinforme } \\
\text { TMMS24 }\end{array}$ & $\begin{array}{c}\text { Procesamiento y tabulación } \\
\text { de resultados con } \\
\text { herramientas de } \\
\text { estadística descriptiva }\end{array}$ & $\begin{array}{c}36 \text { estudiantes del } \\
\text { programa en una sola } \\
\text { aplicación }\end{array}$ & $\begin{array}{c}\text { Objetivo específico 2: } \\
\text { Identificar percepciones sobre } \\
\text { las CSE de los profesores en } \\
\text { formación del PFP. }\end{array}$ \\
\hline $\begin{array}{l}\text { Protocolo de } \\
\text { entrevistas }\end{array}$ & $\begin{array}{l}\text { Entrevistas en profundidad / } \\
\text { Método comparativo } \\
\text { constante } \\
\text { (Strauss y Corbin, 1998) }\end{array}$ & $\begin{array}{c}5 \text { entrevistas en } \\
\text { profundidad a estudiantes } \\
\text { del programa. }\end{array}$ & $\begin{array}{c}\text { Objetivo específico 3: } \\
\text { Categorizar las percepciones } \\
\text { (representaciones, teorías, } \\
\text { supuestos, creencias) sobre } \\
\text { las CSE de los profesores en } \\
\text { formación en función de su rol } \\
\text { en la construcción identitaria } \\
\text { profesional. }\end{array}$ \\
\hline
\end{tabular}

3.3 Instrumentos de recolección de información: Análisis documental, Instrumento de autoinforme y entrevistas

Para realizar el análisis curricular de los programas del PFP, se confeccionaron y aplicaron fichas de registro. Estas fichas de análisis documental y de información se elaboraron en base al marco conceptual de Inteligencia Emocional propuesto por Mayer y Salovey (2011) y Bisquerra (2019) y contemplan los siguientes apartados: una descripción bibliográfica o área de identificación; en segundo momento, una tabla de análisis de contenido temático, espacio que permitió identificar la presencia de conceptos y clasificarlos según frecuencia y temáticas en coherencia con el marco teórico. Finalmente, un espacio destinado al análisis semántico y de las relaciones bajo el modelo de la "interrogación del documento" de Teun A. van Dijk. En su conjunto, la ficha permitió la descripción del contenido como también la extracción y jerarquización de los términos más significativos. La muestra seleccionada correspondió a todos los programas de la carrera.

El TMMS, es un instrumento de autoreporte para medir la Inteligencia Emocional percibida. Su objetivo es explorar los procesos introspectivos de las personas, que se basan en la percepción que tienen sobre sus propias habilidades emocionales.

Salovey et al. (1995) construyeron el instrumento Trait Meta-Mood Scale (Escala Rasgo de Metaconocimiento de los Estados Emocionales, TMMS) para determinar la percepción que los individuos tenían sobre sus habilidades para atender, discriminar y reparar los estados emocionales propios.

Se entenderá por Atención: la capacidad de sentir y expresar los sentimientos de forma adecuada. 
Claridad: la capacidad de comprender bien los estados emocionales propios y Reparación: la capacidad de regular los estados emocionales correctamente.

En su versión original de 48 ítems, se solicita a la persona que evalúe mediante una escala de tipo Likert de 5 puntos (en que 1 representa la opción "Nada de acuerdo" y 5 representa la opción "Totalmente de acuerdo"); el grado de acuerdo con cada uno de los reactivos referidos a tres dimensiones claves en la IE; atención a los sentimientos, claridad emocional y reparación de las emociones.

La capacidad predictiva del TMMS-48 ha sido ampliamente demostrada, siendo sus subescalas las que alcanzaron las mejores propiedades psicométricas evidenciadas por una consistencia interna de entre 0,73 y 0,82 según alfa de Cronbach; en comparación con otras medidas de autoinforme (Davies et al., 1998). En España, se realizó una adaptación al castellano del TMMS-48 evidenciándose similares propiedades psicométricas que las de la escala original inglesa (Álvarez et al., 2019).

Fernández-Berrocal realizó una versión reducida y modificada denominada TMMS-24 que mantiene la misma estructura que tenía la escala original de 48 ítems, consistente en las dimensiones Atención, Claridad y Reparación Emocional; aunque los factores fueron depurados y ciertos ítems de la escala se eliminaron por distintos motivos (Extremera y Fernández-Berrocal, 2004; Angulo y Albarracin, 2018). Algunos, debido a su baja confiabilidad y escasa contribución a la confiabilidad total de la escala general en su versión en español y otros, porque no evaluaban la inteligencia emocional intrapersonal en sentido estricto, sino que, más bien, medían habilidades emocionales interpersonales y aspectos emocionales más generales. (Álvarez et al., 2019). Por estas razones, en el presente estudio se utilizó esta escala.

El Instrumento de autoinforme se aplicó en el mes de noviembre de 2020, en un solo momento y en el contexto de una clase a la que asiste todo el grupo, enviando previamente toda la información del proceso y la invitación voluntaria a participar. Cada uno de los encuestados completó un consentimiento informado y respondió en un periodo no superior a los 20 minutos. Las respuestas fueron anónimas.

Las entrevistas aplicadas fueron de tipo semiestructuradas (Escudero, 2018) con categorías, objetivos y secuencia preestablecida, fueron grabadas y transcritas una vez aplicadas las pautas de entrevista a 5 estudiantes seleccionados bajo el muestreo de variación máxima (Escudero, 2018). Los criterios utilizados fueron: tipo de especialidad, tipo de dependencia administrativa del centro de práctica y experiencia como docente en el sistema educativo.

Las entrevistas fueron analizadas mediante el método de la comparación constante de datos propuesto por Corbin y Strauss (2002). Esto permitió codificar y analizar los datos de forma simultánea para desarrollar conceptos que permitieron la comprensión del fenómeno de estudio. Este procedimiento se desarrolló en cuatro etapas: la primera implica la comparación de los datos recolectados (codificación abierta); la segunda supone una integración de cada categoría con sus propiedades (codificación axial); la tercera requiere delimitar la teoría que comenzará a desarrollarse (codificación selectiva); y por último, en la cuarta etapa, que se produce tras un proceso de saturación de los incidentes pertenecientes a cada categoría, se levanta la teoría comprensiva del fenómeno de estudio.

Finalmente, se cautelaron criterios de rigor y consistencia cualitativa (Vasilachis, 2019). Primero, la credibilidad sustentada a partir de las evidencias obtenidas de los participantes del estudio. Segundo, un proceso de auditabilidad tanto de los instrumentos como de los resultados obtenidos entre los diferentes integrantes del equipo de investigación y un experto externo. Finalmente, la triangulación de los resultados obtenidos por medio de la concurrencia de información de diferente naturaleza.

\section{Resultados}

\subsection{Resultados análisis documental}

La información obtenida de la aplicación de las fichas de análisis documental y de información fue analizada por medio del análisis de discurso, el que, según Van Dijk (1980), corresponde a 
un conjunto de procedimientos analíticos de información verbal obtenida de diversas fuentes. Su operacionalización se hizo siguiendo el modelo de macroestructuras de Van Dijk (1980; citado en Peña y Pirela, 2007), que refiere a la determinación de los trozos de texto claves a través de los cuales se puede representar el contenido de los documentos analizados, asumiendo que este contenido es lo que con mayor seguridad recordarán los individuos de la puesta en contacto con alguna de estas fuentes de información. Lo anterior se realizó en base a la construcción de tablas de frecuencia y jerarquización de términos que permitieron rastrear la presencia, frecuencia y predominancia de las habilidades socioemocionales en los programas de estudio.

Un primer resultado obtenido corresponde a los términos frecuentes que aparecen de manera explícita en los programas. Las categorías que más se repiten, tanto en los programas de asignaturas de la línea de pedagogía general como en las didácticas específicas corresponde a los conceptos de autoevaluación (entendida en relación con otras personas), coevaluación, metacognición, aprendizaje colaborativo, empatía, resolución de problemas, autoconfianza académica y personal.

Un segundo resultado corresponde a la presencia y predominancia de las dimensiones del nivel de conocimiento y regulación que los sujetos tienen sobre sus propias emociones: atención, claridad y reparación. La predominancia se observó respecto de la dimensión reparación, la cual se presentó en todos los programas analizados (didáctica, fortalecimiento disciplinar y asignaturas de la línea de pedagogía general). A su vez, en las asignaturas de fortalecimiento y asignaturas de la línea de pedagogía general se evidenció en segundo lugar de predominancia la presencia de la dimensión de claridad. Sin embargo, se pudo establecer que no hay presencia de la dimensión atención en los programas analizados.

Respecto de la sección del programa en la cual las CSE se presentaron, ya sea de manera explícita o implícita, estas fueron referidas en la redacción de los "Resultados de Aprendizaje", mientras que en las CSE que se encontraron en la sección "Metodología" fueron siempre implícitas recurriendo a los términos "trabajo grupal", "autoevaluación", o "coevaluación". Cabe señalar que del total de programas revisados, en el 10,7\% de estos hubo ausencia total de las CSE, tanto en un nivel implícito como explícito.

\subsection{Resultados de la aplicación del instrumento de autoinforme TMMS24}

Los resultados de la escala de medición TMMS24 se agrupan de acuerdo con los tres aspectos medidos: atención, claridad y reparación. Cada uno de estos aspectos está categorizado en tres niveles: bajo/debe mejorar, adecuado y demasiado o excelente, dependiendo del aspecto. Cada nivel está diferenciado por sexo.

Debido a que, en este estudio, el instrumento de autoinforme se realizó en forma anónima, ya que para el cumplimiento de los objetivos planteados este aspecto no era relevante de distinguir, no fue posible hacer una diferenciación de los resultados por sexo, por lo que se toma la decisión de trabajar con intervalos completos sin diferenciación por sexo, quedando entonces las categorías de la siguiente manera:

\section{Tabla 2.}

Categorías emergentes y puntajes del TMMS 24

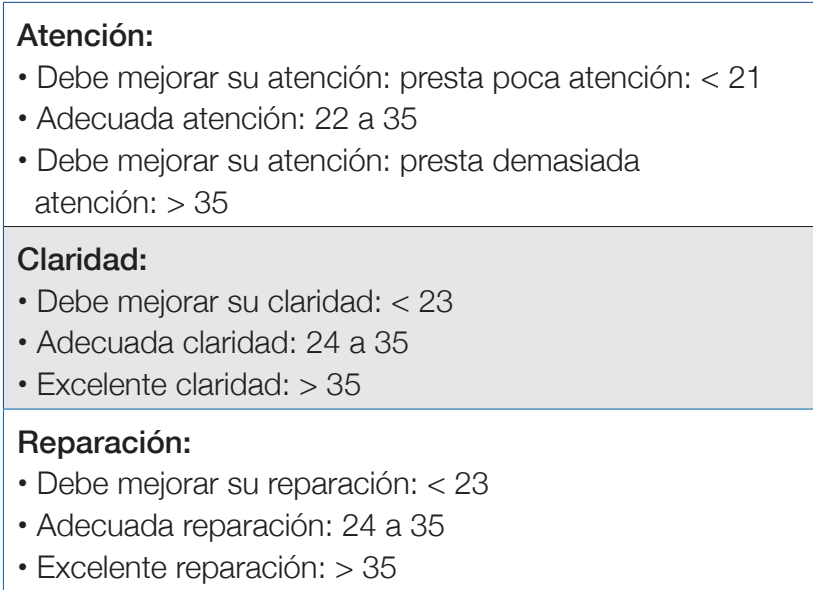

Los resultados del autoinforme por aspecto muestran que la mayoría de los participantes percibe una adecuada atención, claridad y reparación emocional. Destaca, por ejemplo, que cerca de un 90\% de los participantes perciba tener adecuada o excelente claridad, y más de un $86 \%$ declara algo similar para el aspecto de reparación emocional. Sin embargo, un porcentaje importante de los 
participantes (37\%) percibe que presta demasiada atención a sus emociones, lo cual se considera deficiente respecto a las directrices de la escala.

\section{Figura 1:}

Puntuación en TMMS24 aspecto Atención

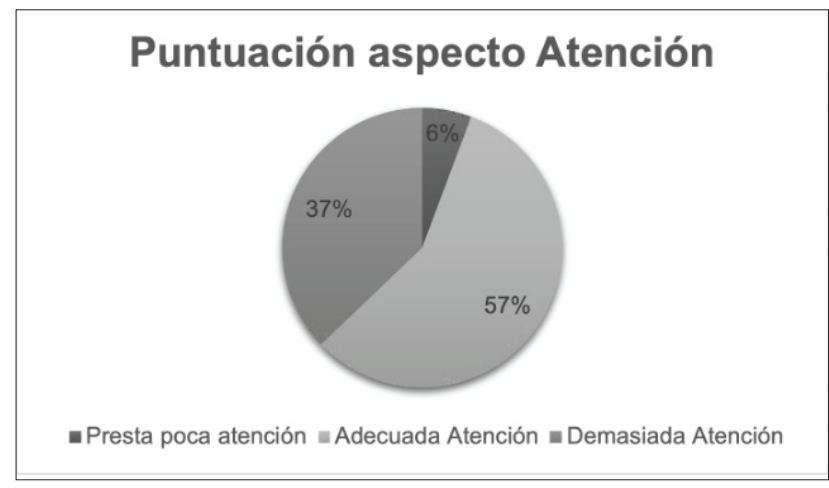

\section{Figura 2:}

Puntuación en TMMS24 aspecto Claridad

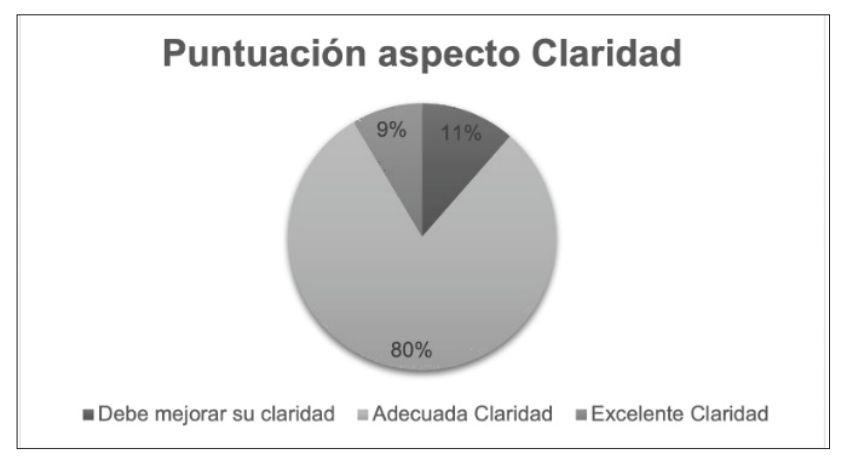

\section{Figura 3:}

Puntuación en TMMS24 aspecto Reparación

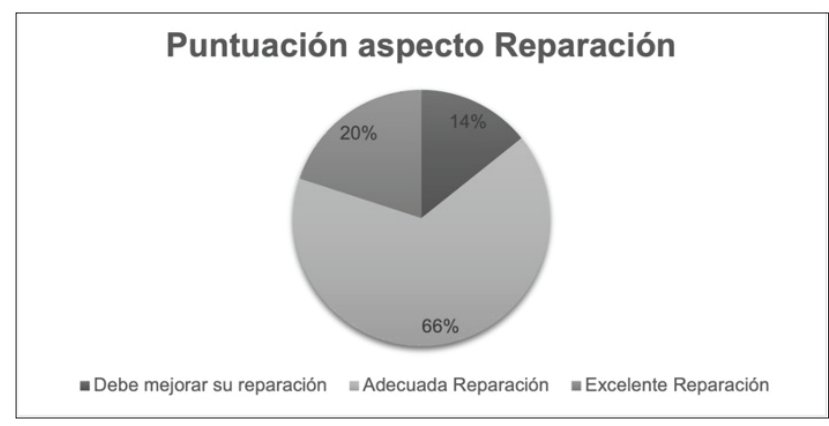

Un segundo ámbito de análisis de los resultados fue el desglose por cada afirmación del instrumento de autoinforme, de la frecuencia de respuestas. Con el fin de tener más información específica sobre las respuestas de los participantes, se calculó la frecuencia de respuestas para cada afirmación del test por aspecto. En este nivel de detalle se observan las afirmaciones correspondientes al aspecto de atención con las que los participantes concuerdan más, tales como: Afirmación 1: "Presto" mucha atención a los sentimientos" en la cual más de un 96\% está bastante, muy o totalmente de acuerdo; Afirmación 4: "Pienso que merece la pena prestar atención a mis emociones y estado de ánimo, con la cual más de un 97\% está bastante, muy o totalmente de acuerdo"; Afirmación 8: "Presto mucha atención a cómo me siento", con la cual poco más de un $91 \%$ está bastante, muy o totalmente de acuerdo. $Y$ con respecto al aspecto claridad, la Afirmación 13: "Normalmente conozco mis sentimientos sobre las personas" muestra que más del $71,43 \%$ de los encuestados reporta tener claridad sobre lo que siente respecto de los otros. En resumen, del análisis de las frecuencias por afirmación se concluye que en la categoría de Atención es donde se encuentra una mayor concordancia de percepciones entre los participantes y que apuntan a tener una alta atención a su emocionalidad, lo que refuerza lo planteado en el análisis por categorías.

\subsection{Resultados entrevistas en profundidad}

Después de aplicado el análisis cualitativo a las entrevistas mediante el método comparativo constante (Corbin y Strauss, 2002), se pudo obtener dos categorías de datos verbales, a saber: 


\section{Figura 4:}

Familia de códigos "Habilidades socioemocionales en profesores en formación"

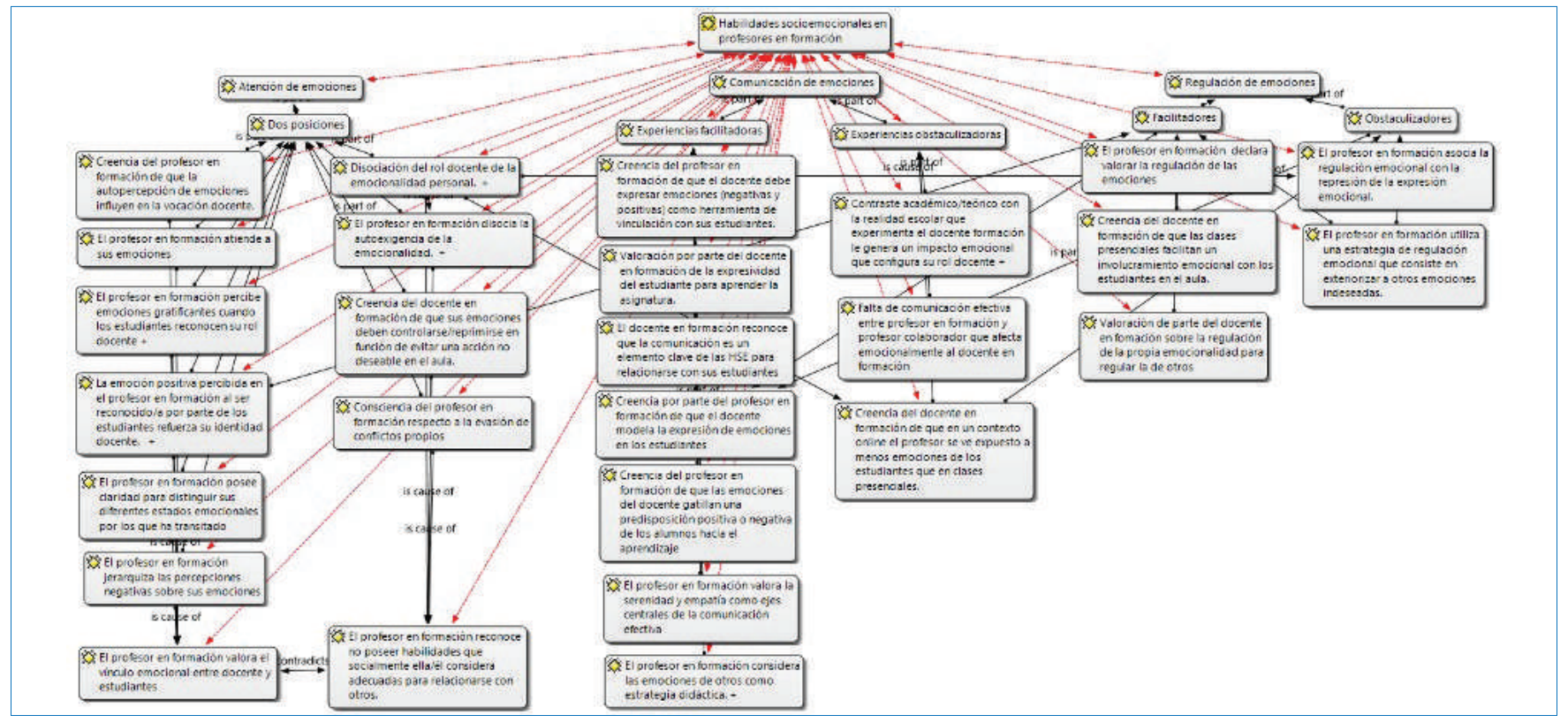

De acuerdo a la figura 4, esta categoría surge a partir de testimonios respecto a las distintas CSE desplegadas durante la formación práctica, las cuales se agruparon en tres subcategorías:

Atención de emociones: aquí se pudo hallar la presencia de dos posiciones diferentes respecto a cómo se han ido atendiendo las emociones por parte de los entrevistados. En la primera posición, algunos profesores mencionaron que sí atendían a sus emociones, así como las de sus estudiantes, porque asumen como premisa que el reconocimiento de sus emociones es parte del quehacer docente y se refuerza en el momento en que sus estudiantes en el aula valoran el rol del docente. Al respecto, la siguiente cita menciona lo siguiente:

Ahí cachai de nuevo entran las emociones también en un plano más personal, no solamente por la disciplina, por lo que estay enseñando, sino en la relación con los chiquillos y las chiquillas, o sea, si a mí me importan mis estudiantes y yo les importo a ellos ${ }^{3}$ ahí se vuelve más fácil, la conversación, o sea porque uno escucha a alguien que le importa cachai...(Estudiante PFP 4_H).

En cambio, en la segunda posición se destaca el rol que posee la represión de emociones del docente en función de evitar situaciones indeseables en el aula, tal como se menciona a continuación:

Eehh... visualizo dos situaciones, una en la que el entusiasmo y la entretención sean muy grandes... a mí me gusta mucho el humor negro y la ironía, entonces con este humor uno pasa a llevar mucho las líneas que limitan como los márgenes de cada circunstancia y si es que me dejo llevar por esto, podría empezar a bromear con no sé, amenazas de notas y cosas así, solo con el motivo de querer trastocar la ley (Estudiante PFP 5_A).

Comunicación de las emociones: En este ámbito se presentan dos tipos de experiencias, aquellas facilitadoras y otras obstaculizadoras del proceso de expresión de emociones por parte de los profesores en formación. En el primer caso, se agrupan creencias que surgen a partir de vivencias de los profesores en formación, las que relevan la importancia de la comunicación como un elemento 
clave para el desarrollo de los estudiantes en el ámbito socioemocional. Uno de los profesores en formación plantea:

Yo no estuve ahí para acercarme a conversar en ese momento y eso a ella le golpeó cachai, lógicamente, o sea... y claro, como que eso da cuenta de lo importante que es cómo comunicarse y ser transparente también en la comunicación... quizás si yo le hubiera dicho " a ver, sabí que, yo no puedo entrevistarme contigo todas las semanas, pero cada vez que tu hables con el psicólogo del colegio yo sé lo que está pasando", o sea, si yo le hubiese transmitido, transparentado quizás, pero al no estar esa comunicación, ehhm, ella se sintió decepcionada, cachai? y fue clave que me lo dijera también... de nuevo un rol esencial de la comunicación, sino yo nunca me hubiese dado cuenta. (Estudiante PFP 4_H).

En el segundo caso, confluyen elementos que durante la práctica dificultaron la comunicación, tales como un alto contraste entre lo académico y lo teórico, falta de comunicación entre el docente colaborador y el profesor en formación, así como dificultades para abordar el proceso educativo en ambientes virtuales. Uno de los profesores en formación puntualiza el siguiente obstáculo:

Sí, yo creo que es una limitante en la cantidad de emociones que una se ve expuesta durante todo este tiempo. Yo creo que todavía no me he visto expuesta a la cantidad de emociones que yo creo que voy a sentir en el minuto de estar frente a una clase. En el fondo, este es mi tiempo de preparación y si yo tengo algún problema puedo apoyarme de la profesora colaboradora, preguntarle a la universidad. El otro año, que ya voy a estar trabajando voy a estar sola frente a la vida, enfrentándome por primera vez a esas emociones que por esta situación se limitaron. (Estudiante PFP 1_I).

En relación con la regulación de las emociones, también es posible encontrar aspectos facilitadores y aspectos obstaculizadores. De esta manera, los profesores en formación declaran valorar la regulación de las emociones, para algunos es necesario la regulación de la propia emocionalidad para potenciar esta CSE en sus propios estudiantes, pero como contrapartida, esta regulación emocional se asocia con la represión de la expresión de los estados emocionales personales.

En estos casos podemos apreciar la valoración de la regulación de la propia emocionalidad como un elemento facilitador y en el caso de los elementos obstaculizadores es relevante considerar que, junto con la represión de la expresión emocional, el profesor en formación disocia su propia emocionalidad de su rol docente.

\section{Figura 5:}

Familia de códigos "Presencia de habilidades socioemocionales en la identidad docente"

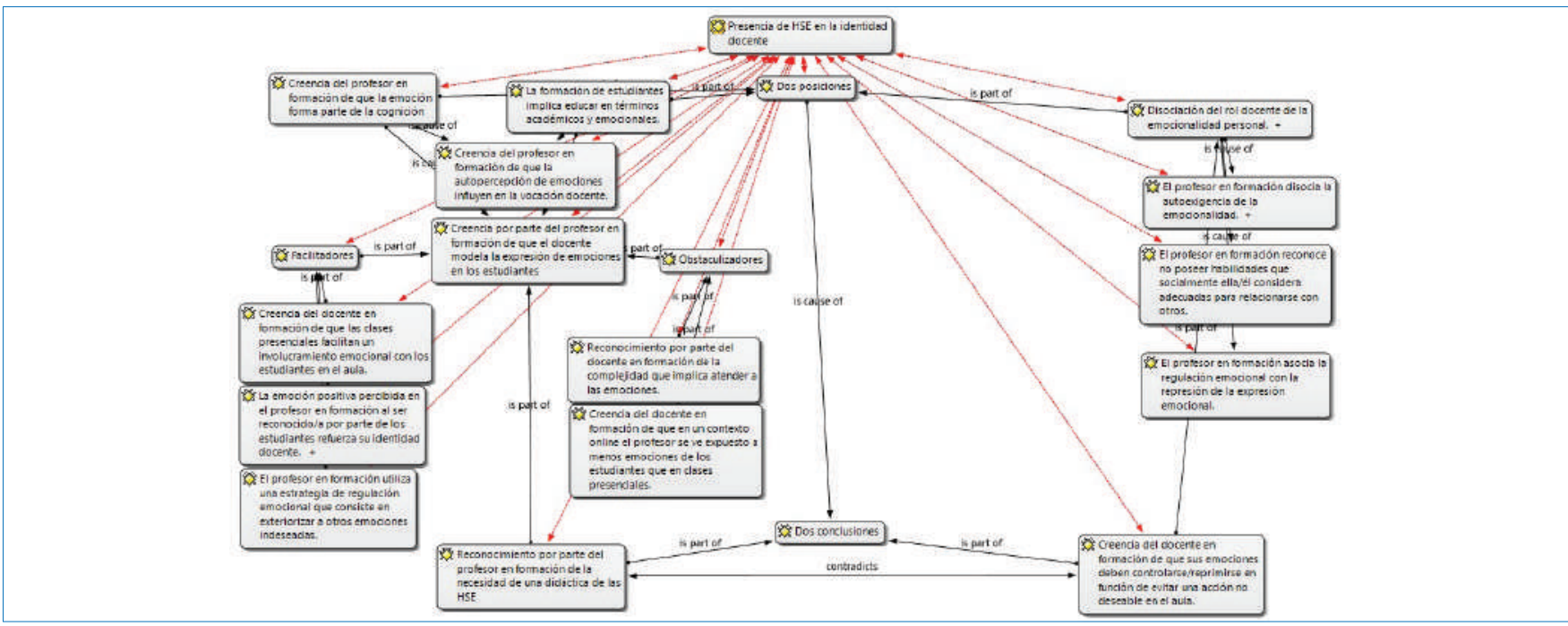


Se observan dos grandes posiciones. La primera refiere a la creencia por parte del profesor en formación de que la emoción forma parte de la cognición. En este sentido, las emociones conviven con la cognición en su ejercicio profesional y como elementos asociados en la labor docente.

....te cuentan cosas que no le cuentan a sus papás o a sus amigos, porque bueno es su familia o sus pares y uno está ahí en un lugar tan intermedio que muchas veces ven como una válvula de escape....entonces si uno no quiere estar ahí no vay a poder entregar, o sea, yo creo que lo más problemático de ser profesor es que es 7 profesiones en 1, o sea, nuestra pega no se termina cuando uno termina de hacer la clase de Guerra Fría y es súper importante, súper importante que los profesores sepan mucho de su disciplina, etc., pero siento que estamos principalmente acompañando a niños, niñas, a jóvenes por casi la mitad de su día por muchos años... entonces estamos ahí ciertamente más que para hacer una clase. (Estudiante PFP 4_H).

La segunda posición que se aprecia en la familia de códigos explicita la disociación entre el rol docente y la emocionalidad personal, ya que existe una serie de factores que obstaculizan un vínculo entre ambos elementos, tal como se puede apreciar en el siguiente fragmento de algunos de los entrevistados:

Yo no tengo tanta tolerancia a la frustración, es una emoción que a mí me cuesta. Me he descargado mucho con mi marido, en ese sentido, descarga emocional y esa sensación de injusticia, eso es lo que

\section{Figura 6:}

Modelo comprehensivo o teoría emergente sobre las habilidades socioemocionales en los profesores en formación.

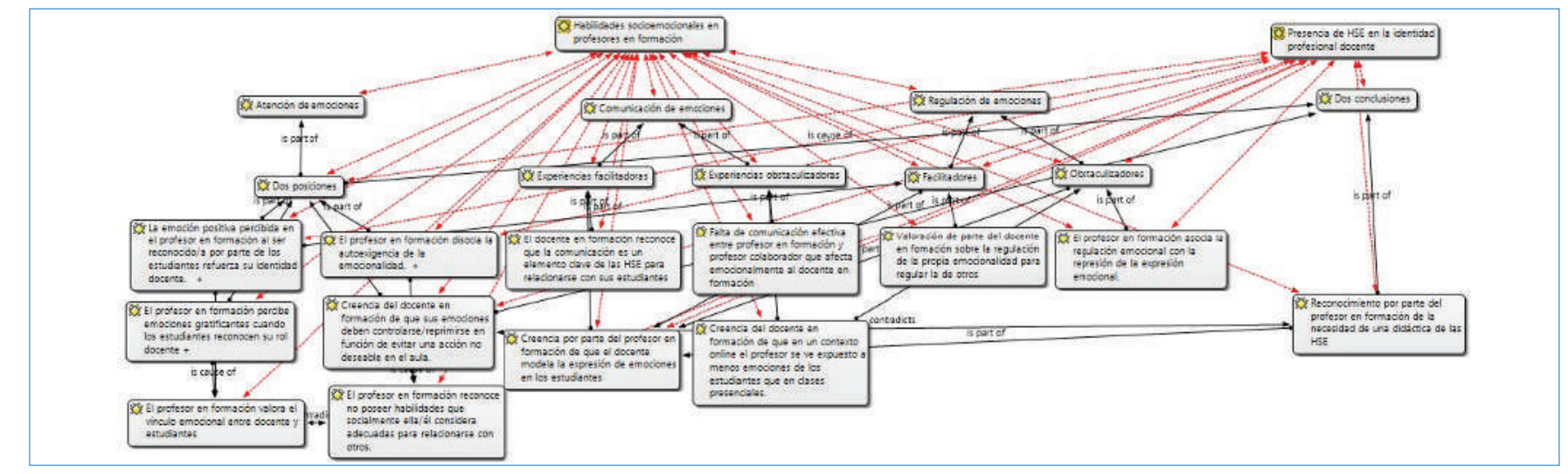

me pasa. Eso lo descargo con la gente cercana, en este caso mi marido y a través de eso he logrado, desde esa emoción que descargo, sacar cosas positivas para poder transmitirlo. (...) Sí, de todas maneras, yo con ella tengo que ser un siete (refiriéndose a la docente colaboradora), no puedo expresarle de repente estas frustraciones, esta angustia, este sentimiento de impotencia (Estudiante PFP 1_I).

En relación con la primera posición presentada, los profesores en formación presentaron una serie de elementos favorecedores y obstaculizadores para modelar la expresión de emociones en sus estudiantes. Dentro de los elementos que facilitan esta práctica se encuentran aquellas creencias relacionadas con el rol que cumplen las clases presenciales en generar un mayor involucramiento emocional, la exteriorización de emociones y el reconocimiento positivo de su rol por parte de los estudiantes. Algunos de estos elementos se aprecian en el siguiente texto:

La segunda clase me salió mucho mejor y todo. $Y$ cuando las niñas me empezaron a decir Madamme porque estoy en la Maisonette, entonces como que dicen Madame. (...) Demasiado feliz, porque me sentí como una profesora verdadera (Estudiante PFP 2_M).

Respecto a los obstaculizadores se aprecian las dificultades propias de una docencia online o remota en la que el contexto presencial estaba completamente ausente producto de la pandemia por Covid-19. 
El modelo presentado corresponde a la teoría emergente producto del análisis de las entrevistas realizadas, y donde en particular se evidencian relaciones ya existentes entre características de las categorías de información previamente presentadas. La relación más destacada es la existencia de dos posiciones respecto a la presencia de las CSE en la identidad profesional de los profesores en formación. Una, que realza estas como parte de las competencias que el docente debe aprender para promoverla en sus estudiantes, y que, en consecuencia, debiera estar contemplado como parte del currículum de los programas de formación inicial. En particular en la línea de didáctica asociada a esta temática, lo cual es reconocido por algunos participantes. Al respecto, destacamos el siguiente extracto de una de las entrevistas para exponer la necesidad de una "didáctica de las emociones":

Estudiante PFP4_H: han pasado millones de cosas y que yo nunca pensé que iba a tener que lidiar... y el mostrarse como una persona que siente también, ha sido esencial como... pero también, no en esa perspectiva "Ah si yo entiendo perfecto lo que te pasa". porque no entiendo perfecto lo que les pasa a chiquillas que les ha pasado cosas que a mí nunca me han pasado. Sí, claro. Y eso es lo difícil, yo encuentro... porque lo encuentro fundamental pero no sé si uno teniendo una clase en que te hablen de la importancia de las emociones, que es importante, cierto, como uno pueda transmitirle a alguien "Ah sí, esta debería ser yo" ... es difícil esa relación, encuentro yo.

Luego, el/la participante destaca en el transcurso de la entrevista respecto del rol de las emociones en sus decisiones pedagógicas, aspecto muy ligado al código anterior, el docente en formación reconoce:

Las cosas pasan porque las personas tienen ideas, porque las personas sienten cosas, porque a las personas les pasan cosas, etc... y en ese sentido, las emociones es super importante de transmitir eso. A ver... "La gente tenía rabia" ya por qué tenía rabia?, bueno, porque las condiciones de vida eran inhumanas, porque los sueldos eran insuficientes, etc... y visibilizar eso, que son personas que tienen emociones y que esas emociones los movilizan... como... entonces en eso, yo creo que hay un momento de la práctica pedagógica las emociones ahí tienen un rol como... pero de nuevo, súper desde mi disciplina, yo no sé cómo alguien de Física podría hacerlo cachai... pero porque mi disciplina es una disciplina de seres humanos que actúan en base a emociones igual que nosotros, les quitaban el $90 \%$ de su sueldo, ¿Cómo te sentirías tú si te quitan el 90\% de tu sueldo?, no pucha, me daría lata, ¿Me daría lata? o ¿Qué haría y con esa lata?, no pucha.. cachai? eso es como fundamental, es justamente para insertarse que es algo que yo creo que nos han permitido avanzar desde una forma de enseñar que no es tan contenidista sino de comprender las cosas que pasan... (Estudiante PFP 4_H).

Sin embargo, existe también una posición discordante sobre lo anterior, sostenida por elementos obstaculizadores vividos por algunos de estos docentes durante su formación, que da cuenta de la disociación entre CSE de la formación docente en general, sosteniendo entonces que estas competencias no debieran estar contempladas en el ejercicio de la docencia en aula, al punto de incluso reprimir o evitar expresarlas ante los estudiantes.

Un segundo elemento constitutivo de la teoría emergente, producto de la relación entre las categorías ya presentadas, es la presencia de las CSE de los docentes en formación en dos de sus componentes teóricos: la atención de las emociones y la regulación de las emociones, ambas vividas de manera diferente entre los participantes de acuerdo con sus experiencias vividas. Ahora bien, un hallazgo que emergió del análisis realizado es la aparición de un componente no contemplado como tal en la definición de estas competencias, correspondiente a la comunicación de emociones, la cual se valora por los participantes de manera transversal, aunque con connotaciones disímiles entre sí al haber experiencias facilitadoras como obstaculizadoras para su desarrollo durante la formación docente vivida. En este sentido, para algunos se visualiza como una oportunidad para el proceso de enseñanza - aprendizaje y como una necesidad profesional no resuelta para otros. 


\section{Discusión}

Todos los instrumentos aplicados evidenciaron la falta de explicitación de las CSE en el currículum y particularmente su inexistencia en los programas de estudio de las asignaturas. Esto concuerda con los diagnósticos revisados en la teoría previa y que fueron realizados en diversos países y programas (Waajid et al., 2012; Nenonene, 2019). Tal como plantean Zahonero y Martín (2012) respecto del desarrollo de las CSE:

Tradicionalmente, profesores de todos los ámbitos educativos han ido adquiriendo estas competencias de un modo autodidacta, intuitivo, y centrado en la experiencia. Evidentemente la falta de preparación sólida en este terreno, se ha reflejado con demasiada frecuencia en los procesos educativos. Por ello consideramos que debieran ser incluidas dentro de los currículum de los programas de formación del profesorado (p.61).

En la misma línea, y gracias a las entrevistas, se pudo recoger la necesidad de desarrollar una didáctica de las competencias socioemocionales como se aprecia en los códigos: "El docente en formación identifica las CSE como elemento de la didáctica específica de su subsector/El profesor en formación considera las emociones de otros como estrategia didáctica". Como vimos en la sección anterior, esta necesidad se vincula directamente con el área curricular que se enseña: "porque mi disciplina es una disciplina de seres humanos que actúan en base a emociones igual que nosotros" (PFP-4_H). Todo esto concuerda con los hallazgos relacionados con el análisis documental realizado a los programas de asignatura del programa de formación pedagógica. En efecto, tanto las entrevistas como el análisis documental relevan, tanto implícita como explícitamente, este vacío formativo.

En relación con el instrumento de autoinforme, los resultados obtenidos permiten afirmar que el grupo de profesores en formación muestra una alta autopercepción en cada una de las dimensiones, demostrando que consideran que las emociones son aspectos a los cuales atienden, conocen y manejan permanentemente. Sin embargo, se aprecia un exceso de preocupación por el aspecto de atención, donde estarían actuando preferentemente por la atención a su emocionalidad, más que a un equilibrio con los aspectos de razonamiento. Del mismo modo, parece que el aspecto menos desarrollado, aunque siempre dentro de lo esperado en la mayoría, es la reparación, o sea, la capacidad que tendrían los sujetos de regular su emocionalidad. Lo anterior refuerza nuevamente la imperiosa necesidad de enseñar las CSE en el trayecto formativo docente que arrojó la investigación y que fue planteado por la teoría previa. Zahonero y Martín (2012) ya habían señalado que:

Cuando actuamos como profesores, desempeñamos nuestra labor trabajando con los niveles de competencia socioemocional que, con más o menos presencia de lo intuitivo, hemos ido acumulando a lo largo de nuestro proceso de socialización (...) tener estos procesos presentes resulta, pues, fundamental, ya que en muchos casos algunas de nuestras limitaciones, (cuando no carencias), como docentes, reposan en la ausencia o precariedad en la formación de ámbitos que debieran ser básicos en las competencias docentes (p.61).

La adecuada percepción que los estudiantes manifestaron en este instrumento de autorreporte se sustenta en el supuesto de que las respuestas fueron dadas con honestidad y bajo un satisfactorio nivel de autoconocimiento, pero también podrían estar teñidas por factores como la deseabilidad social y un bajo nivel metacognitivo en torno a la propia emocionalidad. Estos resultados validan el diseño metodológico que permite la profundización y matización de estos datos con los otros instrumentos usados en la investigación: entrevistas de profundización y análisis documental, lo cual está en línea con lo que reporta GutiérrezCobo et al. (2019). Por otro lado, si bien los profesores en formación manifiestan comprender las emociones (claridad), si consideramos los resultados del test, esto no se condice con lo expresado en las entrevistas en profundidad, en las que no se explicitan experiencias que puedan fundamentar claridad. Ahora bien, sí se evidencian en las entrevistas las dimensiones de atención y reparación emocional.

Estas dos últimas dimensiones adquieren características diversas a partir del análisis efectuado de las entrevistas. Así las cosas, ambos componentes presentan elementos 
favorecedores y obstaculizadores en el contexto del proceso formativo del profesor en formación de este programa de formación pedagógica. Esto da cuenta de que las percepciones sobre las competencias socioemocionales no se presentan de forma monolítica, como podría apreciarse si consideramos solamente los resultados del autorreporte al contestar el TMMS24, sino que estas percepciones son más complejas si tenemos en cuenta las experiencias vividas y las creencias reforzadas por estas experiencias que fueron relatadas por los profesores en formación.

Asimismo, la construcción de un ethos profesional se ve permeada tanto por experiencias facilitadoras como obstaculizadoras, aspecto que también ha sido destacado en otras investigaciones que estudian los procesos de formación docente (Schagen et al., 2017). Se trata de una tensión entre las experiencias nuevas en relación con la formación docente y las creencias que cada profesor en formación traía consigo (Borg, 2009). Esto evidencia la complejidad propia de la construcción de la identidad profesional que implica un proceso delicado de posicionamiento tanto como profesor de aula y como profesor en formación. Lo anterior nos permite sustentar una de las propuestas que se pudo levantar a partir de la investigación, la necesidad de incluir la enseñanza explícita de los aspectos socioemocionales en los programas de formación docente y su vínculo con el desarrollo de la identidad profesional. En el transcurso de la construcción de la identidad profesional influyen diversos factores entre los cuales las emociones y habilidades socioemocionales cumplen un rol fundamental. Tal como planteó Schutz (2014) "esta construcción y reconstrucción de sus identidades se basa no solo en el constante cambio del conocimiento de sí mismos, sino también en los cambios constantes respecto de la percepción que tienen de su profesión" (p.173). Considerando que los resultados nos arrojaron un grupo de estudiantes que no consideraba importante la IE en su desarrollo profesional, es importante incluir estas temáticas y discusiones dentro de la trayectoria formativa para que puedan cambiar la percepción sobre la labor educativa.

Un componente emergente en esta investigación lo constituye la categoría comunicación de las emociones. En efecto, se trata de un rasgo saliente que el modelo comprehensivo resultante recoge luego de los análisis efectuados a partir de las entrevistas realizadas. Así, la comunicación es un elemento clave no solo para la expresión de las emociones, sino que también para el ejercicio docente en el aula de enseñanza media.

\section{Conclusiones}

Esta investigación tuvo como objetivo analizar las percepciones sobre las CSE de los profesores en formación del PFP en la construcción de la identidad profesional con el objeto de informar las trayectorias formativas. Para esto, se desarrolló un análisis documental que permitió describir el contexto de la formación docente y analizar el grado de presencia de estas habilidades en el material curricular. También, se identificaron y categorizaron estas percepciones. Primero, a través de un test validado que nos permitió relevar que los profesores en formación valoran y reconocen la importancia de las CSE y reportan un alto grado de comprensión de las emociones. En este punto, aparece como hipótesis posible a estudiar la intencionalidad de los participantes en las respuestas al Instrumento de autorreporte, que parecen estar centradas en su percepción personal y no en relación al rol que realizan (docentes), lo cual concuerda con estudios anteriores.

Como segundo punto, las entrevistas en profundidad permitieron ampliar la identificación y categorización de percepciones, las que a partir de los sucesivos procesos de análisis y codificación relevaron dos aspectos centrales: habilidades socioemocionales en profesores en formación y presencia de habilidades socioemocionales en la identidad docente, los cuales a su vez posibilitaron la identificación y categorización de diferentes percepciones y creencias. Estas percepciones se relacionan con la atención, la regulación y la comunicación de emociones, por un lado y, por otro lado, con dos tipos de posicionamiento frente al rol que les cabe a las habilidades socioemocionales en la conformación de la identidad docente. Asimismo, el aspecto claridad que la literatura reconoce y que el instrumento TMMS24 contempla dentro de sus dimensiones apenas emerge del discurso de los profesores en formación. 
Nuevas direcciones asociadas al campo de conocimiento se relacionan con profundizar la estrecha relación entre la cognición y la emoción, así como la forma en que ambas se vinculan en la formación de profesores de enseñanza media.

Las proyecciones prácticas que emergen a partir de los resultados son concordantes con el objetivo $\mathrm{N}^{\circ}$ 4 de nuestra investigación y consisten en propiciar una mayor colaboración entre los docentes del programa, incorporar un pilar teórico común referido a las CSE en las didácticas, entregar oportunidades de formación profesional para que integre estas competencias. En el ámbito curricular, propiciar una actualización de los programas de asignaturas y la correspondiente explicitación de las CSE en los instrumentos curriculares e incorporación de estas habilidades en el diseño de clases considerando enfoques múltiples y diferencias disciplinarias en estrecha relación con las didácticas de las disciplinas y que permitan construir una plataforma común, un lenguaje básico en relación con la regulación de las emociones y con las estrategias de manejo de conflicto, entre otros. Así contribuimos a enseñar lo tradicionalmente omitido en educación superior.

\section{Referencias bibliográficas}

Álvarez, J., Hurtado, A. y Hurtado, M. (2019). Competencias socioemocionales en estudiantes de la carrera de pedagogía en educación diferencial especialidad discapacidad intelectual de la Universidad SEK. [Tesis de Magíster en Docencia Universitaria, Universidad SEK]

Angulo, R. y Albarracín, A. (2018). Validez y confiabilidad de la Escala rasgo de metaconocimiento emocional (TMMS-24) en profesores universitarios. Revista Lebret, 10, 61-72.

Angouri, J. (2010). Quantitative, qualitative or both? Combining methods in linguistic research. En L. Litosseliti (Ed.), Research Methods in Linguistics (pp. 29-45). Continuum.

Ávila, N. y Cortés, A. (2017). El género" informe de caso" en la formación inicial docente: una aproximación basada en la actividad. Lenguas Modernas, (50), 153-174.
Bisquerra Alzina, R. y Mateo, J. (2019). Competencias emocionales para un cambio de paradigma en educación. Editorial Horsori.

Borg, S. (2009). English language teachers' conceptions of research. Applied linguistics, 30(3), 358-388.

Cassullo, G. y García, L. (2015). Estudio de las Competencias Socio Emocionales y su Relación con el Afrontamiento en Futuros Profesores de Nivel Medio. Universidad de Murcia Revista electrónica interuniversitaria de formación del profesorado, 18(1), 213-228.

Corcoran, R. y Tormey, R. (2010). Teacher education, emotional competencies and development education. Procedia Social and Behavioural Sciences, n. 2, 2448-2457.

Corbin, J. y Strauss, A. (2002). Bases de la investigación cualitativa. Técnicas y procedimientos para desarrollar la teoría fundamentada. CONTUS Editorial Universidad de Antioquia.

Creswell, J. W. y Creswell, J. (2018). Research design. Qualitative, quantitative, and mixed methods approaches (5th ed.). SAGE Publications Inc.

Davies, M., Stankov, L. y Roberts, R. D. (1998). Emotional intelligence: In search of an elusive construct. Journal of Personality and Social Psychology, 75(4), 989-1015.

Escudero, C. y Cortez, L. (Coords.) (2018). Técnicas y métodos cualitativos para la investigación científica. Editorial UTMACH

Extremera, N. y Fernández-Berrocal, P. (2004). La importancia de desarrollar la inteligencia emocional en el profesorado. Revista Iberoamericana de Educación, 34(3), 1-9.

Fernández Berrocal, P. y Extremera Pacheco, N. (2005). La Inteligencia Emocional y la educación de las emociones desde el Modelo de Mayer y Salovey. Revista Interuniversitaria de Formación del Profesorado, 19(3), 63-93. 
Fragoso-Luzuriaga, R. (2015). Inteligencia emocional y competencias emocionales en educación superior, ¿un mismo concepto? Revista iberoamericana de educación superior, 6(16), 110-125.

Grewal, D. y Salovey, P. (2005). Feeling Smart: The Science of Emotional Intelligence: A new idea in psychology has matured and shows promise of explaining how attending to emotions can help us in everyday life. American scientist, 93(4), 330-339.

Gutierrez-Cobo, M. J., Cabello, R., RodriguezCorrales, J., Megias-Robles, A., GómezLeal, R. y Fernández-Berrocal, P. (2019). A Comparison of the Ability Emotional Intelligence of Head Teachers with School Teachers in Other Positions. Frontier in Psychology, 10, 841. doi: 10.3389/fpsyg.2019.00841

Hirshberg, M. J., Flook, L., Enright, R. D. y Davidson, R. J. (2020). Integrating mindfulness and connection practices into preservice teacher education improves classroom practices. Learning and Instruction, 66 (January 2019), 101298.

Kelchtermans, G. y Deketelaere, A. (2016). The emotional dimension in becoming a teacher. In: LOUGHRAN J. y HAMILTON, M. L. (Eds.), International Handbook of Teacher Education. Volume 2. Dordrecht: Springer. p. 429-461.

Kimura, Y. (2010). Expressing emotions in teaching: Inducement, suppression, and disclosure as caring profession. Educational Studies in Japan, vol. 5, p. 63-78.

Mayer, J. D., Salovey, P., Caruso, D. y Cherkasskiy, L. (2011). What is emotional intelligence and why does it matter? En R. J. Sternberg y J. Kaufman (Eds.), The handbook of intelligence (3rd ed.) (pp. 528-549). Cambridge University Press.

Mertens, D. (2019). Research and evaluation in Education and Psychology: Integrating diversity with quantitative, qualitative, and mixed methods (5th ed.). Thousand Oaks: Sage.
Nenonene, R. L., Gallagher, C. E., Kelly, M. K. y Collopy, R. M. B. (2019). Challenges and Opportunities of Infusing Social, Emotional, and Cultural Competencies into Teacher Preparation: One Program's Story. Teacher Education Quarterly, 46(4), 92-115.

O'Connor, K. (2008). "You choose to care": Teachers, emotions and professional identity. Teaching and teacher education, 24(1), 117126.

Peña Vera, T. y Pirela Morillo, J. (2007). La complejidad del análisis documental. Información, cultura y sociedad, 16, 55-81.

Pérez, J. (2003). Sobre la validez de constructo de la inteligencia emocional. Encuentros en Psicología Social, 1(2), 252-257.

Raspall, L. (2019). Neurociencias para educadores. Homo Sapiens Ediciones.

Reio, T. (2005). Emotions as a lens to explore teacher identity and change: A commentary. Teaching and Teacher Education, 21(8), 985-993.

Salovey, P., Mayer, J. D., Goldman, S., Turvey, C. y Palfai, T. (1995). Emotional attention, clarity, and repair: Exploring emotional intelligence using the Trait Meta-Mood Scale. En J. W. Pennebaker (Ed.), Emotion, disclosure, and health (pp. 125-154). American Psychological Association.

Schagen, A., La Paro, K. y Crosby, D. (2017). Early practicum experiences: Preservice early childhood students' perceptions and sense of efficacy. Early Childhood Education Journal, 45(2), 229-236.

Schutz, P. y Lee, M. (2014).Teacher emotion, emotional labor and teacher identity. Utrecht Studies in Language and Communication, 27(1), 169-186.

Timostsuk, Š, I. y Ugaste, A. (2010). Student teachers' professional identity. Teaching and teacher education, vol. 26, n. 8, p. 1563-1570. 
Van Dijk, T. (1980). Macrostructures. An interdisciplinary study of global structures in discourse, interaction, and cognition. LEA.

Van Dijk, T. (1983). La ciencia del texto. Paidós.

Vasilachis, I. (2019). Estrategias de investigación cualitativa. Vol II. Gedisa.

Vieira, C. (2011). As dificuldades de tomada de decisão vocacional e o auto-conceito em alunos do $8^{\circ}$ e $9^{\circ}$ ano de escolaridade. Tesis (Maestría en Psicología de Educación e Intervención) - Universidade Fernando Pessoa, Porto.

Vivas, M., Chacón M. y Chacón E. (2010). Competencias socioemocionales autopercibidas por los futuros docentes. Educere, 14(48), 137-146.

Waajid, B., Garner, P. W. y Owen, J. E. (2013). Infusing Social Emotional Learning into the Teacher Education Curriculum. International Journal of Emotional Education, 5(2), 31-48.
Wong, C. y Law, S. (2002). The effects of leader and follower emotional intelligence on performance and attitude: An exploratory study. The leadership quarterly, 13(3).

Zahonero Robira, A. y Martín Bris, M. (2015). Formación integral del profesorado: hacia el desarrollo de competencias personales y de valores en los docentes. Tendencias Pedagógicas, 20, 51-70. https://revistas. uam.es/tendenciaspedagogicas/article/ view/2014243-274

Zembylas, M. (2003). Emotions and teacher Identity: A poststructural perspective. Teachers and Teaching, vol. 9, n. 3, p. 213-244. 